American Journal of Applied Sciences 7 (4): 480-485, 2010

ISSN 1546-9239

(C) 2010Science Publications

\title{
Neuroprotective Effect of Quercetin Encapsulated Liposomes: A Novel Therapeutic Strategy against Alzheimer's Disease
}

\author{
${ }^{1}$ W. Phachonpai, ${ }^{2}$ J. Wattanathorn, ${ }^{2} \mathrm{~S}$. Muchimapura, ${ }^{2} \mathrm{~T}$. Tong-Un and ${ }^{3} \mathrm{D}$. Preechagoon \\ ${ }^{1}$ Department of Physiology, Graduate School, Thailand \\ ${ }^{2}$ Department of Physiology, Faculty of Medicine, Khon Kaen University, Thailand \\ ${ }^{3}$ Department of Technology and Pharmaceutical Sciences, Faculty of Pharmaceutical Sciences, \\ Khon Kaen University, Thailand, 40002
}

\begin{abstract}
Problem statement: The prevalence of Alzheimer's disease is increasing but the efficacy of treatment is still very limited due to various factors including the blood brain barrier. Recent findings demonstrated the crucial role of oxidative stress on the pathophysiology of disease. In addition the burden of blood brain barrier can be overcome by nasal administration and vesicle-carrier mediated delivery system. Based on the potent antioxidant effect of quercetin and the burden of blood brain barrier, we hypothesized that the nasal administration of quercetin liposomes could protect against neurodegeneration in Alzheimer's disease. Approach: This study was designed to evaluate the effect and possible action of nasal administration of quercetin liposomes on neurodegeneration in animal model of Alzheimer's disease. Male Wistar rats were pretreated with quercetin liposomes, containing $0.5 \mathrm{mg}$ of quercetin in $20 \mu \mathrm{L}$ via intranasal route once daily continually for 2 weeks before and 1 week after AF64A administration. After the quercetin liposomes treatment, the density of neurons and cholinergic neurons in hippocampus were assessed using histochemical and immunohistochemical techniques whereas the activities of Superoxide Dismutase (SOD), Catalase (CAT), Glutathione Peroxidase (GPx) and Malondialdehide (MDA), a lipid peroxidation product, were determined using biochemical assays. Results: Quercetin liposomes attenuated the degeneration of neurons and cholinergic neurons in hippocampus. The elevation of SOD, CAT and GPx activities and the reduction of MDA in hippocampus were also observed. Therefore, the neuroprotective of quercetin liposomes occurred partly via the reduction of oxidative stress. Conclusion: Our studies suggested that nasal administration of quercetin liposomes may be the potential novel therapeutic strategy against Alzheimer's disease. However, further researches are still essential.
\end{abstract}

Key words: Oxidative stress, Alzheimer's disease, quercetin liposomes, blood brain barrier

\section{INTRODUCTION}

The global prevalence of Alzheimer's Disease (AD) is dramatically increased. It has been estimated that approximate 25 million people worldwide were attacked by AD (Mayeux and Sano, 1999). Although, the etiology of $\mathrm{AD}$ is still unclearly known, free radicals have been recognized to be one important factor (Butterfield, 2004). At present, most of the protective and therapeutic strategies against $\mathrm{AD}$ are still very limited and requires the more effective strategy. Thus, the development of novel therapeutic agent from substances possessing antioxidant activity has been considered.

Quercetin, a flavonoid commonly found in various vegetables and fruits (Chu, 2000). It was reported to protect against oxidative injury and cytotoxicity (Ishige et al., 2001). In addition, oral administration of quercetin was also able to improve learning and memory ability (Wattanathorn et al., 2007). However, the poor absorption and difficulty to pass Blood-Brain-Barrier (BBB) appear to be the big burden for its action particularly in the central nervous system (Boer et al., 2005; Youdim et al., 2004).

Liposomes have long been used as a Drug Delivery System (DDS) to the brain, because the particles can entrap the compounds and prevent rapid elimination or degradation as well as promote penetration through the BBB which in turn decrease the effective dose (Sharma and Sharma, 1997). In addition, they do not elicit negative biological responses that generally occur when a foreign material is introduced in the system. These

Corresponding Author: Jintanaporn Wattanathorn, Department of Physiology, Faculty of Medicine, Khon Kaen University, Khon Kaen 40002, Thailand Tel: 66-43-348394 
lipid vesicles are non-toxic, non-immunogenic, noncarcinogenic, non-thrombogenic and biodegradable (Sinha et al., 2001).

Recently, it had been demonstrated that nasal route, a noninvasive delivery system, could produce higher bioavailability than oral route due to the decrease hepatic metabolism, shorter distance to brain target and easy penetration through the brain (Wang et al., 2006; Illum, 2004). Based on the beneficial effects of quercetin and nasal delivery system, we hypothesized that quercetin liposomes could protect against neurodegeneration in AD. At present, less scientific evidence about this issue is available. Therefore, this study was carried out to evaluate the protective effect of nasal administration of quercetin liposomes against neurodegeneration and its possible mechanism in animal model of AD.

\section{MATERIALS AND METHODS}

Animals: Adult male Wistar rats (180-200 g, 8 weeks old) were obtained from National Animal Center, Salaya, Nakorn Pathom and were housed in group of 6 per cage in standard metal cages at $22 \pm 2^{\circ} \mathrm{C}$ on $12: 12 \mathrm{~h}$ light-dark cycle. All animals were given access to food and water ad libitum. The experiments were performed after the approval of protocol by the Ethical Committee of the Institution and every effort was made to minimize animal suffering in accordance with the internationally accepted principles for laboratory use and care of European Community (EEC directive of 1986; 86/609/EEC).

Experimental protocol: The animals were divided into 5 groups of 8 each as following:

- Liposomes + ACSF: The animals in this group were administered free liposomes via nasal route then they were administered artificial cerebrospinal fluid or ACSF bilaterally via intracerebroventricular route.

- PEG + ACSF: The rats in this group were administered polyethylene glycol or PEG which used as vehicle of quercetin liposomes via nasal route and subjected to ACSF administration.

- Free liposomes + AF64A: All rats were administered free liposomes via nasal route then, they were administered AF64A bilaterally via intracerebroventricular route.

- PEG + AF64A: The animals were administered PEG via nasal route then they were administered AF64A as mentioned in group 3.

- $\mathrm{QCL}+\mathrm{AF64A}$ : The rats in this group were administered quercetin liposomes via nasal route, then they were administered AF64A. The animals were administered the assigned substance at a period of 2 weeks before and 1 week after the administration of AF64A or ACSF.

Preparation and administration of liposomes: Quercetin dehydrate (98\%), high-purity Egg L- $\alpha$ Phosphatidylcholine, Type XVI-E (EPC) and Cholesterol (CHOL) were prepared as quercetin encapsulated liposomes. All animals were administered quercetin liposomes, containing $0.5 \mathrm{mg}$ of quercetin in $20 \mu \mathrm{L}($ dose $=20 \mu \mathrm{g})$, or liposomes without quercetin at the same volume via nasal route with a micropipette at a period of 2 weeks before and 1 week after AF64A or Artificial Cerebrospinal Fluid (ACSF) administration.

AF64A administration: After being anesthetized, the animals were bilaterally administered $(2 \mathrm{nmol} / 2 \mu \mathrm{L})$ AF64A which previously described (Fisher et al., 1982) or ACSF) via intracerebroventricular route according to the following co-ordinates: (from the bregma): posterior $0.8 \mathrm{~mm}$, lateral $\pm 1.5 \mathrm{~mm}$ and ventral (from dura) $3.6 \mathrm{~mm}$.

Determination of neurons density: After 1 week of lesion, they were sacrificed and removed the brains to determine the density of neurons and cholinergic neurons in hippocampus using histochemical and immunohistochemical techniques (Wood and Warnke, 1981).

Biochemical assays: Hippocampal homogenate was prepared in $50 \mathrm{mM}$ phosphate buffer solution $(\mathrm{pH} 7)$ and determined Superoxide Dismutase (SOD), Catalase (CAT) and Glutathione Peroxidase (GPx) activities according to the methods of McCord and Fridovich (1969); Goldblith and Proctor (1950) and Ellman (1959) whereas Malondialdehide (MDA) level were determined using Ohkawa et al. (1979). Protein concentration was measured by Lowry et al. (1951).

Statistical analysis: Results were expressed as mean \pm SEM and determined statistical significance using ANOVA, followed by Duncan's test. The significance was regarded at $\mathrm{p}$-value $<0.05$.

\section{RESULTS}

Both liposomes and PEG had been previously demonstrated to produce no harmful effect on neuron density. The administration of AF64A in either liposomes or PEG treated groups significantly decreased the neuron density in CA1, CA2, CA3 and dentate gyrus ( $p$-value $<0.001$ all; compared to both liposomes + ACSF and PEG + ACSF). Surprisingly, quercetin liposomes administration could reverse these changes ( $p$-value $<0.001$ all; compared to liposomes + ACSF and PEG + AF64A) as shown in Fig. 1. 
Am. J. Applied Sci., 7 (4): 480-485, 2010

Table 1: Effect nasal administration of quercetin liposomes on the activities of SOD, CAT, GPx and the levels of lipid peroxidation in animal model of AD induced by AF64A

\begin{tabular}{lllll}
\hline Experimental conditions & $\mathrm{SOD}\left(\mathrm{U} \mathrm{mg}^{-1}\right.$ protein $)$ & $\mathrm{CAT}\left(\mathrm{U} \mathrm{mg}^{-1}\right.$ protein $)$ & $\mathrm{GPx}\left(\mathrm{U} \mathrm{mg}^{-1} \mathrm{protein}\right)$ & $\mathrm{MDA}\left(\mathrm{U} \mathrm{mg}^{-1} \mathrm{protein}\right)$ \\
\hline Liposomes + ACSF & $16.80 \pm 0.13$ & $18.10 \pm 4.55$ & $14.44 \pm 0.12$ & $1.45 \pm 0.11$ \\
PEG + ACSF & $16.41 \pm 1.27$ & $18.67 \pm 3.84$ & $14.41 \pm 0.11$ & $1.48 \pm 0.17$ \\
Liposomes+AF64A & $11.20 \pm 0.17^{* \#}$ & $16.14 \pm 1.48^{* \#}$ & $11.20 \pm 0.47^{* \#}$ & $2.48 \pm 0.27^{* \#}$ \\
PEG + AF64A & $12.24 \pm 1.90^{* \#}$ & $16.00 \pm 1.73^{* \#}$ & $11.87 \pm 0.22^{* \#}$ & $2.49 \pm 0.26^{* \#}$ \\
QCL + AF64A & $22.50 \pm 0.12^{* \#, \mathrm{a}, \mathrm{b}}$ & $19.80 \pm 3.43^{* \#, \mathrm{a}, \mathrm{b}}$ & $18.31 \pm 0.33^{*, \mathrm{a}, \mathrm{b}}$ & $1.19 \pm 0.13^{* *, \mathrm{a}, \mathrm{b}}$ \\
\hline
\end{tabular}

Results are expressed as mean \pm SEM; ${ }^{*}:$ p-value $<0.001$ compared with liposomes + ACSF treated group; ${ }^{\#}:$ p-value $<0.001$ compared with PEG + ACSF treated group; ${ }^{\text {a }}$ : p-value $<0.001$ compared with liposomes + AF64A treated group; ${ }^{\text {b }}$ : $p$-value $<0.001$ compared with PEG + AF64A treated group

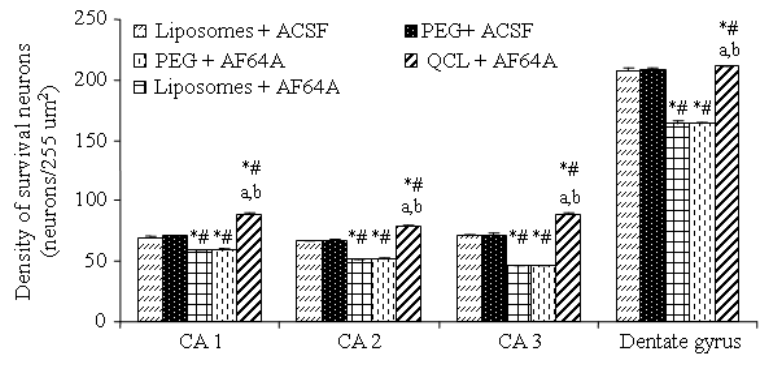

Fig. 1: Effect nasal administration of quercetin liposomes on the density of neurons in hippocampus. $(\mathrm{N}=8)$ Results were expressed as mean \pm SEM. * : p-value $<0.001$ compared with liposomes + ACSF treated group; ${ }^{\#: ~ p-~}$ value $<0.001$ compared with $\mathrm{PEG}+$ ACSF treated group; ${ }^{\text {a. }}$ p-value $<0.001$ compared with liposomes + AF64A treated group; ${ }^{\mathrm{b}}: \mathrm{p}$ value $<0.001$ compared with $\mathrm{PEG}+\mathrm{AF} 64 \mathrm{~A}$ treated group

We also determined the effect of all interventions mentioned earlier on the density of cholinergic neurons in hippocampus as shown in Fig. 2. It was found that quercetin liposomes also reversed the reduction of cholinergic neurons induced by AF64A in all subregions of hippocampus mentioned above.

In order to determine the possible underlying mechanism of quercetin liposomes, we had determined the effect of quercetin liposomes on oxidative stress indices including the level of Malondialdehide (MDA), a product of lipid peroxidation product and the activities of scavenger enzymes including SOD, CAT and GPx in hippocampus as shown in Table 1. Our result showed that both liposomes + AF64A and PEG + AF64A groups generated oxidative stress in rat brain, by decreasing the activities of SOD, CAT and GPx, while increasing the lipid peroxidation. Quercetin liposomes were found most effective in restoring inherent antioxidant system.

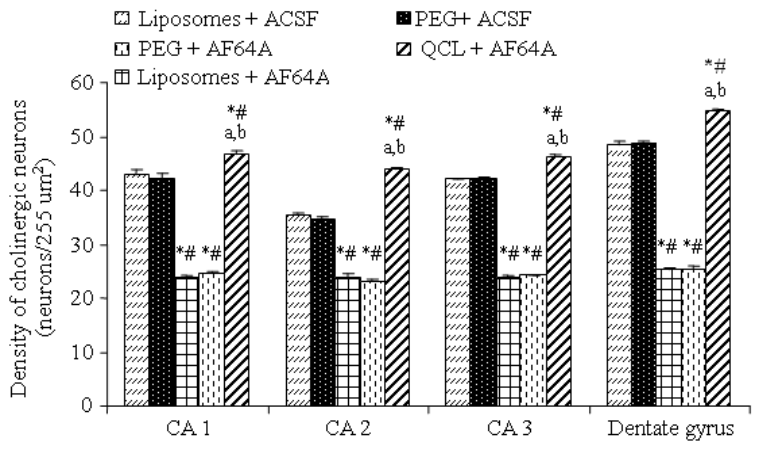

Fig. 2: Effect nasal administration of quercetin liposomes on the density of cholinergic neurons in hippocampus. Results are expressed as mean \pm SEM. ${ }^{*}:$ p-value $<0.001$ compared with liposomes + ACSF treated group; \#: pvalue $<0.001$ compared with $P E G+A C S F$ treated group; ${ }^{\mathrm{a}}$ : $\mathrm{p}$-value $<0.001$ compared with liposomes $+\mathrm{AF} 64 \mathrm{~A}$ treated group; ${ }^{\mathrm{b}}: \mathrm{p}$ value $<0.001$ compared with $\mathrm{PEG}+\mathrm{AF} 64 \mathrm{~A}$ treated group

\section{DISCUSSION}

This is the first investigation that extends available data on nasal administration of quercetin liposomes protective effect against neurodegeneration in animal model of AD. Our results showed that quercetin liposomes significantly increased the survival neurons and cholinergic neurons density in hippocampus and reversed the biochemical alterations seen in hippocampus induced by AF64A.

Various degrees of cognitive impairment were reported to be associated with cholinergic neuronal loss and dysfunction especially in AD (Bondy, 1995). A cholinergic function was previously reported to be required for short-term memory process and the cholinergic dysfunction also played a crucial role for short term memory deficit in AD (Galizia, 1984). Recently, a pile of evidence suggested that the prime candidate responsible for producing the neuronal changes mediating these cognitive deficits appeared to 
be free radicals and oxidative stress generated (Mattson, 2004; Antuti-Castelvetri et al., 2000). Therefore, many studies focused on the beneficial effects of supplement possessing a capability to improve cholinergic function and antioxidant activity were also considered to be a potential candidate for neuroprotective agent against AD.

Quercetin, a main flavonoid found in fruits, vegetables and beverages, was reported to possess antioxidant and cognition (Boots et al., 2008; Reiter et al., 2009). Previous studies confirm that quercetin supplementation improve memory deficit condition induced by reserpine in mice (Naumenko and Kulikov, 2006). However, it was reported that quercetin was easily metabolized after absorption (Manach et al., 2004). Our study found it worthwhile to use the vesicle mediated carrier system particularly liposomes to encapsulated the quercetin against Alzheimer's condition induced by AF64A as well as oxidative stress.

Intracerebroventricular injection by AF64A has been described as an appropriate the substance to destroy the cholinergic system (Hanin, 1996; McDonald and Overmier, 1998). In addition, it could increase oxidative stress in all area of hippocampus, the areas contribution important role on learning leading to the neurodegeneration in the mentioned areas resulting in learning and memory deficit (Gulyaeva et al., 1996) as those observed in $\mathrm{AD}$.

In the present study, the results show that in the AF64A with nasal administration of free liposomes and AF64A with nasal administration of PEG treated groups, there were the reduction of survival neurons and cholinergic neurons in all areas of hippocampus. Moreover, the reduction of scavenging enzymes activities including Superoxide Dismutase (SOD), Catalase (CAT) and Glutathione Peroxidase (GPx), while enhanced the level of Malondialdehyde (MDA), which is an indicative of lipid peroxidation were appear in these groups. These results are in conformity with other workers who have demonstrated cognitive impairment after induced by AF64A in rats (Hanin, 1996; McDonald and Overmier, 1998; Gulyaeva et al., 1996).

No unexpected mortality of any animals occurred after nasal administration of quercetin liposomes used in the present study. As a result, vesicles were considered to be safe at the dosing schedule used. Interestingly, in AF64A with nasal administration of quercetin liposomes treated group, the rats showed significantly improved the density of survival neurons and cholinergic neurons in all areas of hippocampus as compared to the AF64A + PEG or free liposomes treated animals. Moreover, it was notable that the density of cholinergic neurons of quercetin liposomes plus AF64A treated group were higher than those obtained from the liposomes plus ACSF and PEG plus ACSF treated group. This suggests that quercetin liposomes not only the neuroprotective effect but also the neurotrophic effect to stimulate the neurogenesis in hippocampus. Previous study demonstrated that the neurogenesis could occur throughout adulthood especially in hippocampus and subventricular zone of lateral ventricle (Hagg, 2005). Numerous factors had been reported to be regulators of the adult neurogenesis. To clarify this issue, further researches are still required.

Extensive evidence exists for lipid peroxidation being an important mechanism of neurodegeneration in the AD (Liu et al., 2003). Prophylactic treatment with nasal administration of quercetin liposomes significantly reversed the impact of oxidative alterations (MDA, SOD, CAT and GPx) seen in Alzheimer's condition induced by AF64A; this shows the antioxidant potential of quercetin liposomes via nasal administration.

The mechanism how quercetin liposomes could decrease lipid peroxidation and improve its antioxidant systems against Alzheimer's condition induced by AF64A is not known, but it may be presumed that after entering the brain, the quercetin or its derivative might increase the activities of scavenger enzymes mentioned earlier and resulted in the decrease excess free radicals, which in turn decreased the lipid peroxidation process leading to the reduction of MDA as shown in this study. These results are in accordance with the report of quercetin liposomes effect to decrease lipid peroxidation and increase scavenger enzymes activities in rat brain by the induction of cerebral ischemia and reperfusion (Sarkar and Das, 2006). However, the method to prepare the quercetin liposomes and the model to induce the oxidative damage were different.

Our results demonstrated that nasal administration of quercetin encapsulated liposomes was a potential novel strategy to protect against neurodegeneration in hippocampus. It could produce beneficial effect with very much low dose. Based on the previous findings, the decrease effective dose might be associated with many factors including the increase opportunity to transfer directly from the olfactory mucosa along the olfactory pathway to the central nervous system (Cho et al., 2006) via bypassing the Blood-Brain Barrier (BBB), which prevented some CNS-active drugs from reaching the brain (Behl et al., 1998). In addition, it could also decrease the influence of the first pass metabolism (Krauze, 2006). However, the main 
proposed pathway of quercetin delivery via nasal administration was different. It was likely to permeate through the subarachnoid space through the olfactory epithelium and found in the CSF later, because the liposomes behaved as semilipophilic particles. Therefore, quercetin liposomes could rapidly absorb into the CSF. Investigation of quercetin absorption and distribution after administered via nasal route would be of value for future studies.

\section{CONCLUSION}

These results provide experimental evidence for the potential beneficial actions of quercetin liposomes via nasal administration to protect against AD. Further studies are necessary to determine if nasal administration of quercetin liposomes could be a potential strategy for the clinical management of $\mathrm{AD}$.

\section{ACKNOWLEDGMENT}

This study was partial supported by the National Nanotechnology Center (NANOTEC), NSTDA, National Science and Technology Development Agency, Thailand, through its program of Center of Excellence Network.

\section{REFERENCES}

Antuti-Castelvetri, I., B. Shukitt-Hale and J.A. Joseph, 2000. Neurobehavioural aspects of antioxidants in aging. Int. J. Dev. Neurosci., 18: 367-381. DOI: 10.1016/S0736-5748(00)00008-3

Behl, C.R., H.K. Pimplaskar, A.P. Sileno, J. de Meireles and V.D. Romeo, 1998. Effects of physicochemical properties and other factors on systemic nasal drug delivery. Adv. Drug Deliv. Rev., 29: 89-116. DOI: 10.1016/S0169-409X(97)00063-X

Boer, V.C., A.A. Dihal, H. van der Woude, I.C. Arts and S. Wolffram et al., 2005. Tissue distribution of quercetin in rats and pigs. J. Nutr., 135: 1718-1725. PMID: 15987855

Bondy, S.C., 1995. The relation of oxidative stress and hyperexcitation to neurological disease, Proc. Soc. Exp. Biol. Med., 208: 337-345. PMID: 7700883

Boots, A.W., G.R.M.M. Haenen and A. Bast, 2008. Health effects of quercetin: From antioxidant to nutraceutical. Eur. J. Pharmacol., 585: 325-337. DOI: 10.1016/j.ejphar.2008.03.008

Butterfield, D.A., 2004. Proteomics: A new approach to investigate oxidative stress in Alzheimer's disease brain. Brain Res., 1000: 1-7. DOI: 10.1016/j.brainres.2003.12.012
Cho, J.Y., I.S. Kim, Y.H. Jang, A.R. Kim and S.R. Lee, 2006. Protective effect of quercetin, a natural flavonoid against neuronal damage after transient global cerebral ischemia. Neurosci. Lett., 404: 330-335. DOI: 10.1016/j.neulet.2006.06.010

Chu, Y., 2000. Flavonoid content of several vegetables and their antioxidant activity. J. Sci. Food Agric., 80: 561-566. DOI: 10.1002/(SICI)10970010(200004)80:5<561::AID-JSFA574>3.3.CO;2-R

Ellman, G.L., 1959. Tissue sulfhydryl groups. Arch. Biochem. Biophys., 82: 70-77. PMID: 13650640

Fisher, A., C.R. Mantione, D.J. Abraham and I. Hanin, 1982. Longterm central cholinergic hypofunction induced in mice by ethylcholine azirdinium ion (AF64A) in vivo. J. Phamacol. Exp. Ther., 222: 140-145. PMID: 6896342

Galizia, V.J., 1984. Pharmacotherapy of memory loss in the geriatric patient. Drug Intell. Clin. Pharm., 18: 784-791. PMID: 6149111

Goldblith, S.A. and B.E. Proctor, 1950. Photometric determination of catalase activity. J. Biol. Chem., 187: 705-709. PMID: 14803454

Gulyaeva, N.V., N.A. Lazareva, M.L. Libe, O.S. Mitrokhina and M.V. Onufriev et al., 1996. Oxidative stress in the brain following intraventricular administration of ethylcholine aziridinium (AF64A). Brain Res., 726: 174-180. DOI: 10.1016/0006-8993(96)00330-7

Hagg, T., 2005. Molecular regulation of adult CNS neurogenesis: An integrated view. Trends Neurosci., 28: 589-595. DOI: 10.1016/j.tins.2005.08.009

Hanin, I., 1996. The AF64A model of cholinergic hypofunction: An update. Life Sci., 58: 1955-1964. DOI: $10.1016 / 0024-3205(96) 00185-3$

Illum, L., 2004. Is nose-to-brain transport of drugs in man a reality? J. Pharm. Pharmacol., 56: 3-17. DOI: $10.1211 / 0022357022539$

Ishige, K., D. Schubert and Y. Sagara, 2001. Flavonoids protect neuronal cells from oxidative stress by three distinct mechanisms. Free Radic. Biol. Med., 30: 433-446. DOI: 10.1016/S08915849(00)00498-6

Krauze, M.T., J. Forsayeth, J.W. Park and K.S. Bankiewicz, 2006. Real-time imaging and quantification of brain delivery of liposomes. Pharm. Res., 23: 2493-2504. DOI: 10.1007/s11095-006-9103-5

Liu, R., I.Y. Liu, X. Bi, R.F. Thompson and S.R. Doctrow et al., 2003. Reversal of age-related learning deficits and brain oxidative stress in mice with superoxide dismutase/catalase mimetics. Proc. Natl. Acad. Sci. USA., 100: 8526-8531. DOI: 10.1073/pnas. 1332809100 
Lowry, O.H., N.J. Roseburgh, A.L. Farr and R.J. Randall, 1951. Protein measurement with the folin phenol reagent. J. Biol. Chem., 193: 263-275.

Manach, C., A. Scalbert, C. Morand, C. Remesy and L. Jimenez, 2004. Polyphenols: Food sources and bioavailability. Am. J. Clin. Nutr., 79: 727-747. PMID: 15113710

Mattson, M.P., 2004. Pathways towards and away from Alzheimer's disease. Nature, 430: 631-639. DOI: 10.1038/nature02621

Mayeux, R. and M. Sano, 1999. Treatment of Alzheimer's disease. N. Engl. J. Med., 341: 16701679. DOI: $10.1056 /$ NEJM199911253412207

McCord, J.M. and I. Fridovich, 1969. Superoxide dismutase. An enzymic function for erythrocuprein (hemocuprein). J. Biol. Chem., 244: 6049-6055. PMID: 5389100

McDonald, M.D. and J.B. Overmier, 1998. Present imperfect: A critical review of animal models of the mnemonic impairments in Alzheimer's disease. Neurosci. Biobehav. Rev., 22: 99-120. DOI: 10.1016/S0149-7634(97)00024-9

Naumenko, V.S. and A.V. Kulikov, 2006. Quantitative assay of 5-HT (1A) serotonin receptor gene expression in the brain. Mol. Biol., 40: 37-44. PMID: 16523690

Ohkawa, H., N. Ohishi and K. Yagi, 1979. Assay for lipid peroxides in animal tissues by thiobarbituric acid reaction. Anal. Biochem., 95: 351-358. DOI: 10.1016/0003-2697(79)90738-3

Reiter, M., K. Rupp, P. Baumeister, S. Zieger and U. Harreus, 2009. Antioxidant effects of quercetin and coenzyme Q10 in mini organ cultures of human nasal mucosa cells. Anticancer Res., 29: 33-39. PMID: 19331131
Sarkar, S. and N. Das, 2006. Mannosylated liposomal flavonoid in combating age-related ischemiareperfusion induced oxidative damage in rat brain. Mech. Ageing Dev., 127: 391-397. DOI: 10.1016/j.mad.2005.12.010

Sharma, A. and U.S. Sharma, 1997. Liposomes in drug delivery: progress and limitations. Int. J. Pharm., 154: 123-140. DOI: $10.1016 /$ S03785173(97)00135-X

Sinha, J., N. Das and M.K. Basu, 2001. Liposomal antioxidants in combating ischemia-reperfusion injury in rat brain. Biomed. Pharmacotherapy, 55: 264-271. DOI: 10.1016/S0753-3322(01)00060-9

Wang, X., H. He, W. Leng and X. Tang, 2006. Evaluation of brain-targeting for the nasal delivery of estradiol by the microdialysis method. Int. J. Pharm., 317: 40-46. DOI: 10.1016/j.ijpharm.2006.02.055

Wattanathorn, J., W. Phachonpai, A. Priprem and S. Suthiparinyanont, 2007. Intranasal administration of quercetin liposome decreases anxiety-like Behavior and increases spatial memory. Am. J. Agric. Biol. Sci., 2: 31-35. DOI: 10.3844/ajabssp.2007.31.35

Wood, G.S. and R. Warnke, 1981. Suppression of endogenous avidin-binding activity in tissues and its relevance to biotin-avidin detection systems. J. Histochem. Cytochem., 29: 1196-1204. PMID: 7028859

Youdim, K.A., M.Z. Qaiser, D.J. Begley, C.A. Rice-Evans and N.J. Abbott, 2004. Flavonoid permeability across an in situ model of the blood-brain barrier. Free Radic. Biol. Med., 36: 592-604. DOI: 10.1016/j.freeradbiomed.2003.11.023 